\title{
Una mirada a la ingeniería clínica desde las publicaciones científicas
}

\author{
Antonio Miguel Cruz \\ Escuela de Medicina, Universidad del Rosario, Bogotá, D.C., Colombia
}

\begin{abstract}
El objetivo de este trabajo es hacer una caracterización del estado de la ingeniería clínica desde las publicaciones científicas. En primer lugar, se exponen brevemente los factores más significativos que influyen en el cambio y en la evolución de los sistemas sanitarios. Luego, se define el concepto de ingeniería clínica, sus funciones fundamentales y su evolución dentro del desarrollo de los sistemas sanitarios. Finalmente, se expone el estado de la especialidad, dando una mirada desde las publicaciones científicas.

Se puede observar una aparente falta de interés en la comunidad de ingenieros por publicar trabajos de investigación científica. Esto se puede constatar por la franca tendencia al decrecimiento del número de citaciones y el número de publicaciones (en volumen o en cantidad) en las principales revistas del gremio científico.

Finalmente, se exponen, a criterio del autor, los retos actuales y los futuros desarrollos que tiene que enfrentar la especialidad para poder posicionarse en el mundo de las publicaciones.
\end{abstract}

Palabras clave: mantenimiento, evaluación de tecnología biomédica, vigilancia de productos comercializados.

\begin{abstract}
A view of clinical engineering from scientific publications
The aim of this paper was to characterize clinical engineering from the perspective of scientific publications. First of all, the most significant factors that influence the change in health systems are briefly exposed. Then, clinical engineering is defined and its main functions and evolution within the development of the health system is explained. Finally, the author describes the state of the field from the viewpoint of the scientific publications; an apparent lack of interest in the engineering community to publish scientific research was observed.

This behavior can be seen in the clear declining tendency in the number of citations and the number of publications (in volume or quantity) in major scientific journals in the field.

Finally, current challenges and future developments must be addressed to accomplish a better positioning of the specialty in the publishing world.
\end{abstract}

Key words: maintenance; technology assessment, biomedical; product surveillance, postmarketing.

\section{Sistemas sanitarios en un contexto global}

El sistema sanitario es el conjunto de instituciones de un país que se ocupan del estudio, prevención, control y curación de las enfermedades. La sociedad moderna está en una continua búsqueda para incrementar la calidad y mejorar la eficiencia de los sistemas sanitarios, pues se enfrenta constantemente a la introducción de modernas tecnologías, descubrimientos científicos, y cambios demográficos, de regulación y económicos.

La ingeniería clínica, como especialidad, está involucrada en los sistemas sanitarios, pues su

Correspondencia:

Antonio Miguel Cruz, Escuela de Medicina, Universidad del

Rosario, Bogotá, D.C., Colombia.

Teléfono: 347 4570, extensión 257

antonio.miguel@urosario.edu.co

Recibido: 09/06/09; aceptado:18/02/10 razón de ser es ofrecer servicios de apoyo a la tecnología biomédica que se encuentra instalada en ellos. La presencia del ingeniero clínico en el ámbito hospitalario de los países desarrollados surgió por la necesidad de apoyar al sistema médico asistencial en procesos asociados a la adquisición, mantenimiento y control de las mediciones de la tecnología biomédica, así como por la necesidad de lograr incorporar tecnología segura. Por ende, si los sistemas sanitarios evolucionan hacia mejores niveles de prestación de servicios, la ingeniería clínica tendrá que hacerlo también; de lo contrario, estará condenada a desaparecer.

\section{Impacto de los cambios demográficos}

Los cambios demográficos influyen significativamente en la manera en que se ofrecen los servicios de salud. Por ejemplo, en los países desarrollados la población está en franca tendencia al envejecimiento: 
en los Estados Unidos, se espera que para el 2030 el número de personas con 65 o más años de edad represente el $20 \%$;

como la esperanza de vida crecerá a más de 80 años como consecuencia de los avances en la medicina, las enfermedades pasarán de ser fortuitas y repentinas a crónicas.

Estos aspectos implican que los gastos en salud crecerán aún más. Por ejemplo, actualmente ascienden al $60 \%$ del gasto en salud; en el 2020 , se espera que sean del $80 \%$ (1).

\section{Impacto en los cambios de regulación}

Los cambios de regulación son dinámicos y, también, están influyendo significativamente en el sector de la salud. Estos cambios están encaminados hacia dos aspectos fundamentales: por una parte, a reducir los gastos mediante la normalización de los procesos relacionados con el uso de las tecnologías y los procesos asistenciales y, por otra, a aumentar la seguridad del paciente. Por ejemplo, los siguientes aspectos evidenciaron la necesidad de un cambio de regulación y normativo en los países desarrollados:

1. En 1993, un grupo de trabajo en los Estados Unidos reportó al Congreso de esa nación, que se podrían ahorrar cerca de US $\$ 93$ billones de dólares si las organizaciones de salud adoptaran un sistema común de formato de datos para el intercambio de información electrónica sobre los pacientes;

2. En el 2000, el Institute de Medicine de los Estados Unidos informó que, al menos, 98.000 estadounidenses mueren anualmente como resultado de errores médicos. En ese mismo reporte se planteó a la tecnología médica de mayor complejidad como uno de los factores contribuyentes a estas muertes.

Esta última es una de las investigaciones más interesantes que se han realizado, pues en ella se ha llegado a reconocer que

"[...] la tecnología debe ser considerada como un elemento más dentro del sistema médico y dentro de sus roles está el de lograr un mejoramiento del desempeño humano..."(2).

\section{Impacto de los nuevos descubrimientos científicos}

Los nuevos descubrimientos científicos en el campo de la biología y la introducción de las nuevas tecnologías biomédicas, están influyendo de manera dramática en la manera en que se ofrecen los servicios en el sector de la salud. Los tres hechos más significativos que se han producido en los últimos diez años han sido:

1. La decodificación del mapa del genoma humano permitirá identificar los genes "defectuosos" que predispongan a contraer enfermedades. Con una adecuada terapia génica, "reparación del gen dañado", se podrán diseñar nuevos tratamientos. Esto cambiará para siempre el curso de la medicina. La medicina moderna pasará de ser curativa a ser preventiva.

2. Los significativos avances en las investigaciones básicas y aplicadas en el campo de las nanotecnologías provocará que una avalancha de nanodispositivos invada el mercado en los próximos cinco a diez años. Nuevos medicamentos basados en vectores de nanopartículas serán introducidos en el cuerpo humano y repararán el ADN. Los nanosensores, que ya están en etapa de desarrollo, servirán para detectar y regular funciones en los órganos y tejidos; también, se están diseñando nanodispositivos para trabajar en órganos artificiales (3).

3. El avance y desarrollo de las nuevas tecnologías de la información y las comunicaciones prometen jugar un papel fundamental en el sector de la salud. Este desarrollo se manifiesta en dos aspectos fundamentales: el aumento de los niveles de conectividad y la velocidad en el intercambio de datos.

4. El movimiento de las nuevas tecnologías de la información y las comunicaciones, hacia lo que IBM ha llamado o definido como sistemas autónomos (4, Fonash SJ. Micro and nanotechnology: Impact on biomedical science and practice. AAMI Conference, Minneapolis, MN, 2002).

Así como la decodificación del mapa genético y la nanotecnología han revolucionado el campo de la medicina y la construcción de dispositivos electrónicos, lossistemasautónomosrevolucionarán el campo de las ciencias de la computación y de las nuevas tecnologías de la información y las comunicaciones. Estos sistemas invadirán todos los dispositivos en los próximos diez años. Los sistemas autónomos simularán el sistema nervioso central autónomo, se ajustarán a los cambios ambientales, responderán ante ataques externos 
y serán capaces de autorrepararse en caso de fallas.

\section{Impacto de los cambios económicos}

La pregunta fundamental que enfrentan los que deciden sobre políticas de salud en el tema económico, es cuántos recursos destinar a ellas. En otras palabras, se enfrentan a la pregunta: ¿más es siempre mejor? La respuesta intuitiva a esta pregunta es "no". Sin embargo, a pesar de esta respuesta tan evidente, se han efectuado algunas investigaciones para demostrarla con suficiente pruebas científica $(5,6)$.

Los estudios en el campo de lo económico están relacionados con el concepto de los llamados rendimientos marginales decrecientes. Por rendimientos marginales decrecientes se entiende el fenómeno por el cual las unidades adicionales de consumo de atención sanitaria, ya sean de cantidad, calidad o ambas, hacen que los rendimientos (resultados en indicadores de salud) disminuyan o se mantengan constantes a medida que la calidad o calidad de la asistencia sanitaria aumentan. Dicho de otra manera, dar más y más lleva a un punto en el que el beneficio adicional de una unidad monetaria de atención, en cantidad o calidad, no tiene impacto en la salud de la población o de un individuo. De esta manera, se llega al absurdo de que intentar proveer más cantidad o calidad de asistencia sanitaria sólo trae consigo un aumento de los costos, o sea, que la relación beneficio-costo es inferior a la unidad.

Los mencionados estudios (período de análisis: 1960-2002) muestran que la tendencia de la mortalidad infantil (muertes por cada mil nacimientos) y la esperanza de vida al nacer, en comparación con el gasto sanitario como porcentaje del producto interno bruto (PIB) y el gasto sanitario per cápita en España, Portugal y la Unión Europea, no han mejorado significativamente a partir de los años 90 por mucho más que se invierta en salud (5).

Lo interesante y paradójico de la situación relacionadacon losgastos de losserviciossanitarios, es que no son ni los hospitales ni la tecnología los factores determinantes fundamentales en la salud de las personas; sin embargo, es en lo que más se invierte. Ya desde 1980, Kerr Write señaló, luego de un estudio sobre los sistemas sanitarios que, de cada 1.000 personas, 250 no necesitan atención, 740 sólo necesitan atención ambulatoria, 9 requieren de un hospital general y sólo una de un hospital especializado.
Otro estudio realizado en 54 países, produjo dos conclusiones básicas:

1. El nivel de salud de la población está determinado en un país por los siguientes factores, en el orden indicado: educación, vivienda, nutrición, urbanización y recursos médicos (incluida la tecnología).

2. Existe un monto "óptimo" en cuanto a inversiones en el sistema sanitario, a partir del cual sólo se consiguen mejoras marginales en el estado de la salud de la población, que afectan cada vez a menos habitantes. De los estudios realizados en relación con el uso de la tecnología biomédica, se ha comprobado que, si el establecer un diagnóstico con una probabilidad de acierto del 95\% cuesta cinco unidades, para obtener una certidumbre del $96 \%$ es necesario invertir 500 unidades.

Finalmente, un reciente estudio realizado en Europa concluyó que los factores determinantes fundamentales en la salud de las personas son: la herencia, el entorno, los estilos de vida y el sistema sanitario. Su aporte es de $27 \%, 19 \%, 43 \%$ y $11 \%$, respectivamente. Sin embargo, ese mismo estudio encontró que se destina el $90 \%$ del gasto a los sistemas sanitarios (figura 1).

Este fenómeno, relacionado con los gastos destinados a los servicios de salud, no sólo se encuentra en los países de Europa. Por ejemplo, en los Estados Unidos se ha caído en los últimos 10 años en una crisis financiera sin control. Estos hechos pueden resumirse en los siguientes aspectos:

1. En 1997, se gastaba en salud el $13,5 \%$ del PIB, valor muy superior comparado con algunos

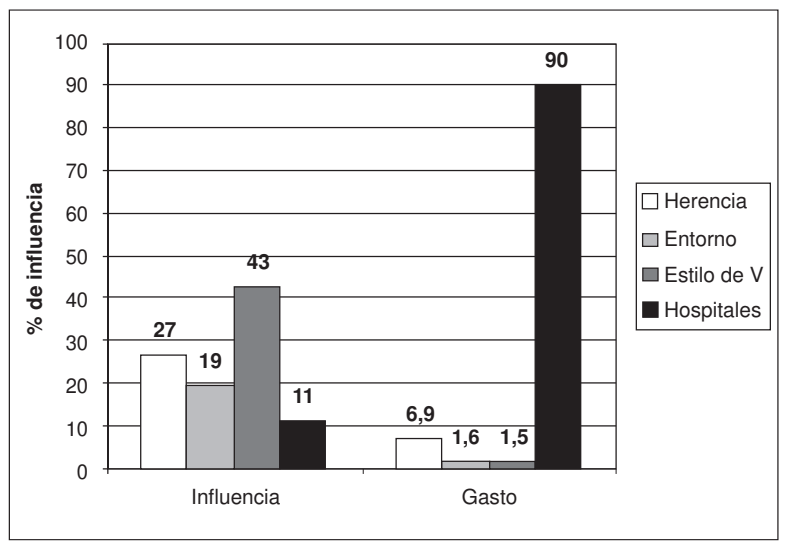

Figura 1. Relación entre factores determinantes y gasto en salud. 
países de la Comunidad Económica Europea, que era del $10 \%$.

2. Los gastos totales crecieron de US $\$ 888$ a US $\$$ 1.425 billones en el 2001. Se espera que ese número alcance los US\$3,1 trillones de dólares para el 2012.

3. Los gastos administrativos están en el orden del $24 \%$. En cambio, estos gastos en Canadá y en la Comunidad Económica Europea están en el orden del $11 \%$ y el $7 \%$, respectivamente.

Estos aspectos han provocado que los estadounidenses, hoy por hoy, paguen más caros los servicios de salud. Paradójicamente, los Estados Unidos no ofrecen cobertura universal de los servicios y tampoco es paradigma en resultados de indicadores de salud. Por ejemplo, en estos momentos ese país se encuentra ubicado en el lugar 37 de 191 países participantes, según una encuesta realizada por la Organización Mundial de la Salud (OMS) relacionada con la calidad de los servicios de salud (7).

Entonces, la pregunta clave no sería, ¿más es mejor?; por el contrario, una pregunta más compleja sería: ¿cuánto gastar para obtener los beneficios esperados? Esto lleva a la conclusión de que es necesario conseguir un monto "correcto" u "óptimo" en el gasto destinado a los niveles de atención en salud.

Existen dos tipos generales de medidas de políticas que intentan afrontar el problema de encontrar un gasto óptimo. La primera es la evaluación de las decisiones, basada en evaluaciones previas, para luego definir las mejores prácticas por seguir para garantizar que los beneficios compensen los costos. En el campo de la economía, esto se suele llamar "evaluación económica".

El segundo tipo de medidas de política económica constituye lo que se denominó "diseño institucional". Este concepto se conoce en la literatura como el diseño adecuado de normas que rigen el comportamiento de los factores que intervienen en el sistema sanitario. Si bien los sistemas sanitarios han tenido un significativo avance en la formulación de las normas que regulan las buenas prácticas en el sistema sanitario, este aspecto por sí solo no es suficiente, pues los que apliquen las normas pueden desvirtuarlas en su cotidiano accionar.

\section{Tecnología en los sistemas sanitarios}

Un sistema sanitario se puede dividir en tres grandes subsistemas: el sistema médico, el sistema médico- administrativo y el sistema médico-tecnológico. Como es obvio, a cada sistema le corresponden aspectos que le son particulares y los cuales deben ser abordados por especialistas en cada disciplina. Así, el sistema médico-tecnológico contribuye de manera significativa en el impacto que tienen los gastos de los sistemas sanitarios. De esta última idea se puede inferir que una adecuada gestión de este sistema contribuiría a mejorar la relación costobeneficio del sistema sanitario en su conjunto.

Las tecnologías biomédicas, como es de suponer, se encuentran en el sistema médico-tecnológico. Según la última definición emitida en Ginebra,

"[...] éstas incluyen los dispositivos, equipos, sistemas, programas, suministros, fármacos, biotecnológicos, así como los procedimientos médicos y quirúrgicos usados en la prevención, diagnóstico y tratamiento de enfermedades en humanos..." (8).

Actualmente, casi toda la comunidad de los profesionales que laboran en los sistemas de salud está de acuerdo en el papel determinante que posee la tecnología en los mismos. A manera de resumen, podemos decir que ésta contribuye a la prevención de enfermedades, así como también permite limitar su impacto. La tecnología es la principal herramienta de diagnóstico para obtener los signos clínicos con el propósito de identificar la naturaleza, la causa y la extensión de un evento no saludable. La tecnología contribuye también al tratamiento por restauración, por mejoramiento o por sustitución de funciones fisiológicas y corporales que estén dañadas, y previene su deterioro o el dolor, garantizándole al individuo el disfrute de una mejor calidad de vida.

La mejor tecnología es aquella que, con gran efectividad en la prevención, detección, análisis y tratamiento de las enfermedades, está también en función del incremento de la esperanza o calidad de vida, de prevenir la muerte prematura, de posibilitar el fácil y rápido acceso a los cuidados de salud, y de incrementar el uso eficiente de los recursos.

Según la Food and Drug Administration (FDA), organismo que se encarga en los Estados Unidos del registro, control y certificación de los dispositivos médicos, en la actualidad existen más de 50.000 tipos diferentes de equipos médicos y cada año se agregan a este arsenal 5.000 nuevos productos.

La explosión en variedad y cantidad de equipos médicos se puede evidenciar por medio de las 
ventas que realizan los países industrializados. Todo esto se debe a una estrecha relación entre la ingeniería y la medicina, que ha dado origen, en los países industrializados, a un gran complejo médicoindustrial, cuya producción alcanzó un mercado por la cantidad de US $\$ 62,5$ miles de millones en 1990. Ésta es la industria de mayor crecimiento proporcional y la que más porcentaje de sus ventas invierte en investigación y desarrollo.

\section{Ingeniería clínica}

La"[...]ingenieríabiomédicaesunadisciplina, que desarrolla y aplica los adelantos de los conocimientos de la ingeniería, la biología y la medicina para mejorar la salud humana a través de la integración de éstas en la práctica clínica..." (8).

La ingeniería clínica se considera una especialidad de la ingeniería biomédica. La inclusión del ingeniero clínico en el ambiente hospitalario surgió por la necesidad de cubrir la brecha existente entre mantener la tecnología en los hospitales y la existencia de los médicos especialistas no entrenados para lidiar con las complejidades de los dispositivos médicos desde el punto de vista técnico.

El nacimiento del término se remonta a los años 60 , cuando César Cáceres lo utilizó por primera vez en 1967. Dos años más tarde obtuvo fondos de la fundación Fannie E. Rippel para crear el primer departamento de ingeniería clínica en un hospital. La existencia de ingenieros clínicos fue formalmente reconocida por la Joint Commission on Accreditation of Healthcare Organizations (JCAHO), en su manual de acreditación del año 1976.

Algún tiempo después, el American College of Clinical Engineering (ACCE) definió al ingeniero clínico como un especialista que

"[...] apoya y contribuye a mejorar la calidad del servicio a los pacientes a través de la aplicación de competencias adquiridas, relacionadas con el campo de la ingeniería y la gestión de la tecnología..." (9).

También, ha sido definido como aquel especialista que

"[...] tiene capacidad de gerenciar sistemas de mantenimiento preventivo y correctivo, adiestrar el personal relacionado con la instalación y realizar pequeños desarrollos, todo ello dentro del ámbito hospitalario con relaciones costo/beneficio óptimas..." (10).
Tradicionalmente, las responsabilidades de los ingenieros fueron creciendo a medida que la tecnología se fue desarrollando y los sistemas sanitarios las fueron adoptando. También, gracias a que los requisitos de regulación fueron evolucionando y exigiendo nuevos retos en la práctica médica y en el manejo de tecnologías.

A manera de resumen, la especialidad ha pasado por los siguientes niveles de complejidad en cuanto a sus funciones, desde los años 70 hasta finales de los 90 .

Nivel 1. Surgió la especialidad y fue reconocida como tal. Se describieron los departamentos recién creados. Se establecieron los primeros programas de control del equipamiento biomédico, que realizaban solamente actividades de reparación y mantenimiento preventivo a equipos de baja complejidad.

Nivel 2. La JCAHO reconoció a la especialidad de Ingeniería Clínica como parte responsable en el cumplimiento de las actividades de mantenimiento preventivo e inspección. Tres años más tarde, en 1979, el Emergency Care Research Institute (ECRI) (organización no gubernamental que trabaja con instituciones de salud en los Estados Unidos) publicó en la Revista Dispositivos de Salud de ese año una adaptación o revisión del programa de control del equipamiento médico. Hizo énfasis, y ahí radicaba lo novedoso, en la necesidad de reportar los daños y los accidentes que ocurren al interactuar los pacientes con tecnologías biomédicas, producto de malos mantenimientos o procedimientos operativos. Se agregó, además, una nueva responsabilidad o función, la cual consistía en que se empezó a prestar atención al proceso de adquisición y a tratar de gestionar íntegramente los contratos de servicios.

Nivel 3. Durante la década de los 80 , los departamentos de ingeniería clínica se empezaron a preocupar por los aspectos económicos. Esto ocurrió principalmente porque la JCAHO emitió en su nueva edición del manual de acreditación de hospitales, la posibilidad de lograr acreditación sin la existencia de un departamento de ingeniería clínica en el hospital. En este nivel surgió la posibilidad de que las instituciones de salud evaluaran el outsourcing como una alternativa.

Los departamentos que "sobrevivieron" a este escollo, comenzaron a responsabilizarse de las actividades de mantenimiento preventivo y correctivo del equipamiento complejo de 
especialidades tales como radiología y laboratorio clínico. Informatizaron el control y la gestión de mantenimiento del equipamiento biomédico.

Nivel 4. Los departamentos asumieron todas las funciones propias de un sistema de gestión tecnológica, debido a que la JCAHO, en su manual para la acreditación de instituciones de salud de la serie "Plan Technology and Safety Management" exigió la creación de un sistema integral de políticas y procedimientos en cuanto a la adquisición de nuevas tecnologías y la sustitución de las ya obsoletas, programas de control del equipamiento biomédico, inspecciones, mantenimiento preventivo y correctivo, reporte de accidentes, y gestión de contratos de servicios. También, exigió el análisis de costos y de productividad, crear metodologías relacionadas con la construcción y renovación del equipamiento, y un programa de control de riesgos en un sistema de aseguramiento de la calidad

Nivel 5. Es el nivel en el cual se debería estar actualmente. El papel del departamento de ingeniería clínica en un sistema de gestión de tecnologías continuará creciendo, especialmente, en la adquisición del equipamiento con los objetivos de satisfacer los estándares internacionales en materia de salud y de que no existan problemas con la adquisición de equipamientos de alto costo, como los de resonancia magnética $(R M)$ y tomografía axial computadorizada (TC).

En resumen, de las dos definiciones anteriores y de la evolución de la especialidad a través de los años 70, 80, y 90 en los países desarrollados, se puede observar un avanzado nivel de desarrollo. Se identifica que la función fundamental del ingeniero clínico es la de diseñar y aplicar los procesos asociados a la gestión tecnológica hospitalaria, cuyo objetivo fundamental es alcanzar una atención de excelencia a costos razonables, esto mediante el empleo racional y eficiente de la tecnología (11).

Ahora bien, un panorama bien diferente existe en los países subdesarrollados y, en especial, en América Latina. Se puede afirmar que la ingeniería clínica como especialidad aún tiene un desarrollo incipiente. Por ejemplo, la Organización Mundial de la Salud anotaba, a finales de la década de los 90 del siglo pasado, respecto a los procesos de dirección, mantenimiento y reparación de equipos utilizados en la salud, que

"[...] un país en desarrollo tiene rara vez el $50 \%$ de sus equipos en estado de utilización y que en algunos casos hasta el $80 \%$ pueden ser inoperables...".

Según este mismo informe, el factor principal que determina esta desastrosa situación es la falta de calificación del personal técnico, médico y paramédico con relación a la

"[...] utilización de nuevas tecnologías y la incapacidad de generar desarrollos en el ámbito médico-hospitalario que puedan elevar la calidad de la atención..." (2).

Con respecto a Colombia, se puede decir que no existe ningún estudio que se haya propuesto como problema de investigación caracterizar completamente el estado real de la ingeniería clínica. Algunos estudios revelan que la situación no es diferente a la del resto de los países de la región. Esto se puede constatar al indagar sobre dos aspectos fundamentales:

1. estudios realizados por investigadores e instituciones, las cuales evidencian dificultades en la gestión de tecnologías, piedra angular de las funciones de un ingeniero clínico, y

2. el estado de desarrollo de los grupos de investigación de ingeniería clínica en Colombia.

Por ejemplo, en relación con el primer punto, hay dos estudios importantes:

- El primero está relacionado con el estado de los procesos asociados a la adquisición de nuevas tecnologías. Éste reveló que, de todas las adquisiciones que se realizan en las instituciones públicas, $34 \%$ se hizo a precios iguales o inferiores a los precios de referencia en el mercado internacional, $22 \%$ se realizó con sobreprecios de hasta el $10 \%$, otro $22 \%$ con sobreprecios entre el $11 \%$ y el $50 \%, 8 \%$ a sobreprecios que oscilan entre el $51 \%$ y el $100 \%$. y finalmente, $14 \%$ con sobreprecios de más del $100 \%(12)$.

- El segundo tiene relación con la gestión de los contratos de servicios de mantenimiento. Éste reveló que

"[...] en Colombia no se han realizado profundos estudios sobre este tema, las únicas indagaciones que se han hecho se refieren en alguna medida a la importancia que esta estrategia tiene entre las Instituciones Prestadoras de Servicios de Salud...." (13).

Con respecto al estado del desarrollo de los grupos de investigación de ingeniería clínica, al consultar 
el sitio Web Scienti de Colciencias (14), se puede encontrar que existen, al menos, 20 grupos de ingeniería biomédica registrados. De ellos, sólo seis grupos atienden una línea relacionada con la ingeniería clínica; ellos son:

- el Centro de Bioingeniería Universidad Autónoma del Caribe, CEBI-UAC (categoría D),

- el Grupo Ciencia y Tecnología Biomédica, CTB, de la Universidad de Antioquia (categoría D),

- el Grupo de Investigaciones en Ingeniería Biomédica, G-BIO, de la Universidad Autónoma de Cali (categoría D),

- el Grupo de Investigación en Ingeniería Biomédica, EIA-CES (GIBEC), categoría B,

- Ingeniería de la Salud de la Universidad Nacional de Colombia (categoría A) y

- el grupo llamado IngeBioCaribe de la Universidad Simón Bolívar (categoría D).

Nótese como el 66,67\% de los grupos que abordan la ingeniería clínica como tema de investigación, poseen un valor bajo en la categoría de impacto (categoría D). Las categorías son A1, A, B, C, D y registrado; la más alta es $A 1$ y la más baja es registrado. Por esta razón, al observar la producción científica en términos de proyectos y publicaciones de estos grupos, se puede decir que es poca y de poco impacto.

\section{Futuro de la ingeniería clínica}

El punto de inflexión (cuando una curva cambia de concavidad, matemáticamente hablando, es cuando hay un cambio de signo en el cero de la segunda derivada de la función), estratégico en una industria, ha sido definido por Andrew Grove, presidente de la Corporación Intel,
"[...] como el momento preciso en el cual fuerzas extremas, externas e internas, cambian para siempre el escenario de la industria en cuestión, creando oportunidades y retos..." (15).

Del modelo o idea de Grove se infiere que, una vez se llega al punto de inflexión o justo antes de llegar a él, la industria debe estar preparada para tomar medidas, evitando así evitar caer en un descenso de su crecimiento. Aplicando este interesante concepto al contexto de la ingeniería clínica, hay quienes han llegado a decir que ésta se encuentra en un punto de inflexión estratégico (1).

Probablemente, los mencionados autores que plantean la existencia de un punto de inflexión para la ingeniería clínica estén en lo cierto. A nuestro modo de ver, el problema ha radicado en que la especialidad ha estado mayormente vinculada al servicio técnico y no ha realizado o no ha profundizado en la realización de investigaciones básicas y aplicadas en el campo de las nuevas tecnologías de la información y las comunicaciones, la investigación de operaciones, la inteligencia artificial, la minería de datos y la gestión económica, para utilizarlas en su propio beneficio. Para poder hacer esto, la especialidad debe pasar por un proceso de maduración más profundo, aumentar su campo de acción, en donde se deben identificar los problemas científicos por resolver, y aumentar la vinculación que tiene que existir entre el sector hospitalario (que sería el símil de la industria) y la academia (en este caso, las universidades).

La mayoría de los autores que argumentan el citado punto de inflexión tienen la opinión -y es la nuestra también- de que la ingeniería clínica debe aumentar el alcance en las investigaciones que realiza. Si se da una mirada a la ingeniería clínica

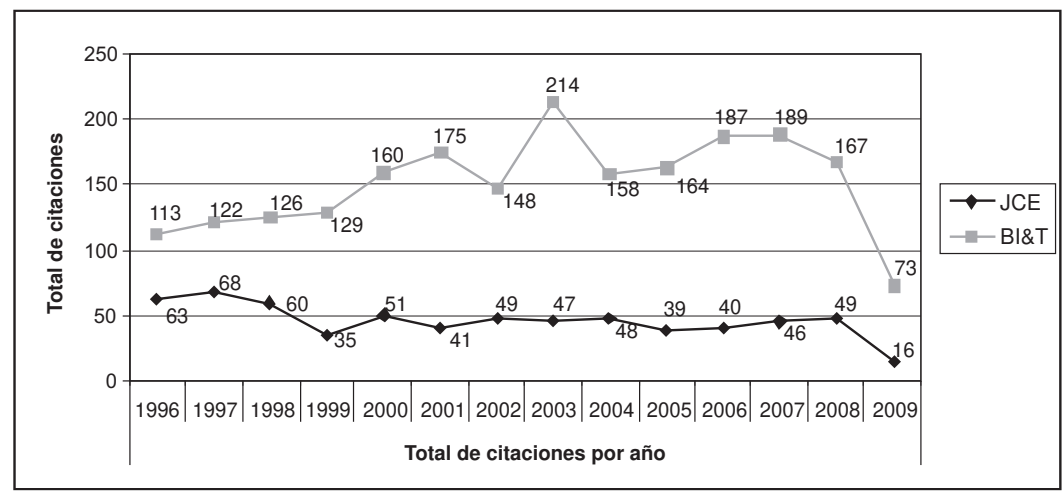

Figura 2. Total de citaciones por año en las revistas Journal of Clinical Engineering y Biomedical Instrumentation \& Technology (16). 
desde la ventana de las publicaciones científicas, se puede observar que los resultados corroboran la opinión anterior. El comportamiento observado se evidencia por igual, tanto en los países desarrollados como en los subdesarrollados.

En esta especialidad, tradicionalmente han existido tres revistas en las cuales se pueden publicar trabajos de investigación. Éstas son:

1. Journal of Clinical Engineering, publicada desde 1976 y cuya casa editorial es Lippincott Williams \& Wilkins,

2. Biomedicallnstrumentation\& Technology,órgano oficial de la Association for the Advancement of Medical Instrumentation (AAMI), publicada desde 1989 por la casa editorial Hanley \& Belfus for the Association, y
3. IEEE Engineering in Medicine and Biology Magazine, publicada desde 1982, por la casa editorial Institute Electrical and Electronics Engineers, Engineering in Medicine \& Biology.

Esta última está registrada en The Web of Science, con un índice de impacto de 1,25 unidades, aproximadamente. La base de datos SCOPUS de revistas indexadas (16) permite estudiar cuatro indicadores para medir el impacto que tienen los artículos publicados en una determinada revista; estos son:

1. el total de artículos publicados por año,

2. el total de citaciones por año,

3. el porcentaje de artículos que nunca se citan, y

4. la línea de tendencia de artículos publicados; este indicador mide la cantidad de citaciones

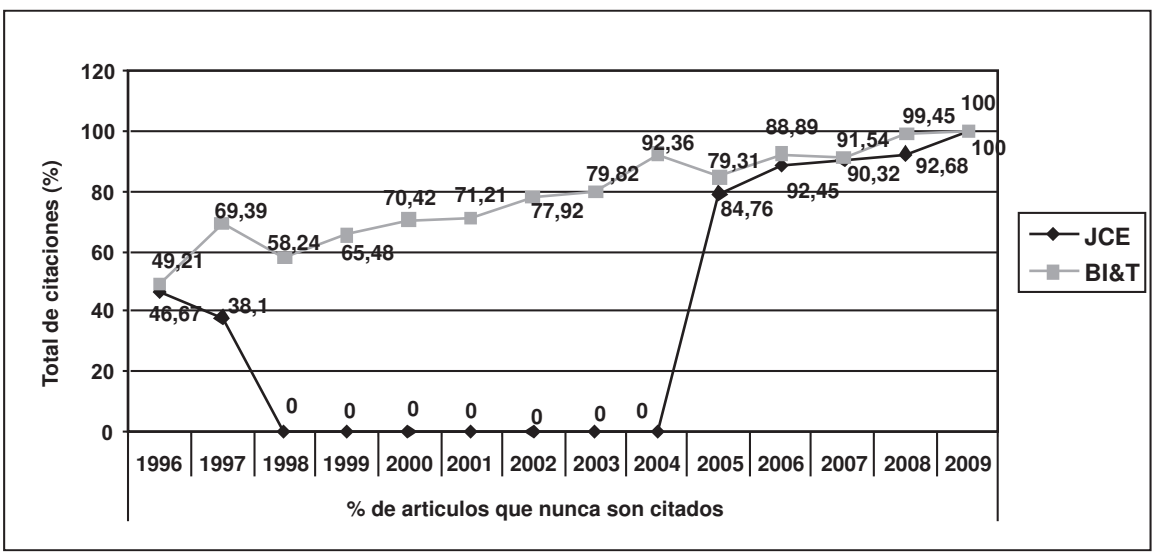

Figura 3. Porcentaje de artículos que nunca son citados en las revistas Journal of Clinical Engineering y Biomedical Instrumentation \& Technology (16).

Nota aclaratoria: la revista Journal of Clinical Engineering dejó de publicar artículos en el periodo 1998-2004.

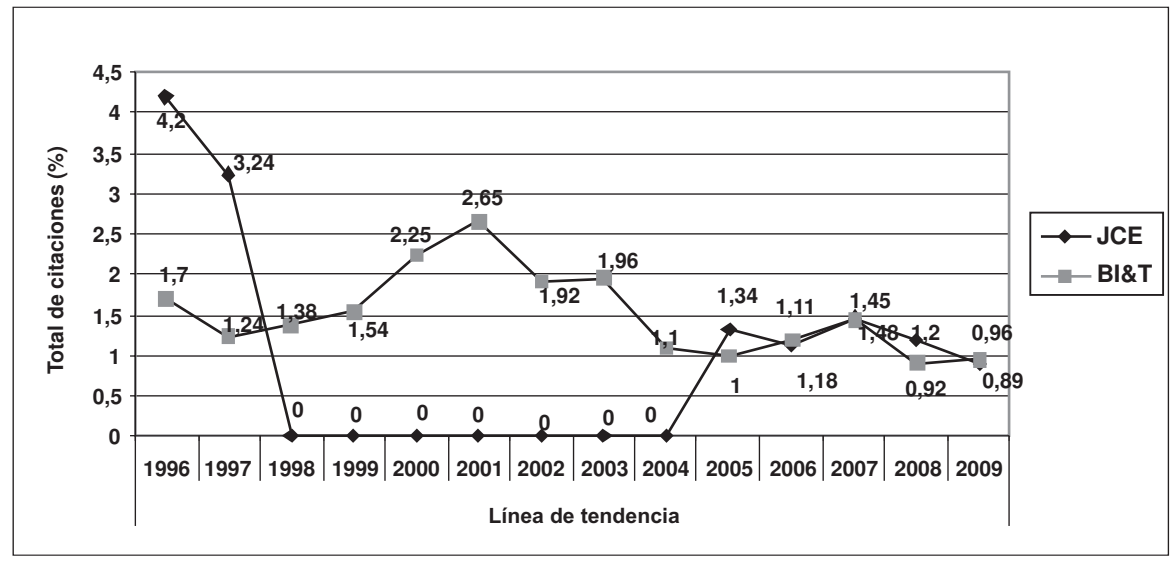

Figura 4. Línea de tendencia del impacto en publicaciones en las revistas Journal of Clinical Engineering y Biomedical Instrumentation \& Technology (16).

Nota aclaratoria: la revista Journal of Clinical Engineering dejó de publicar artículos en el periodo 1998-2004. 


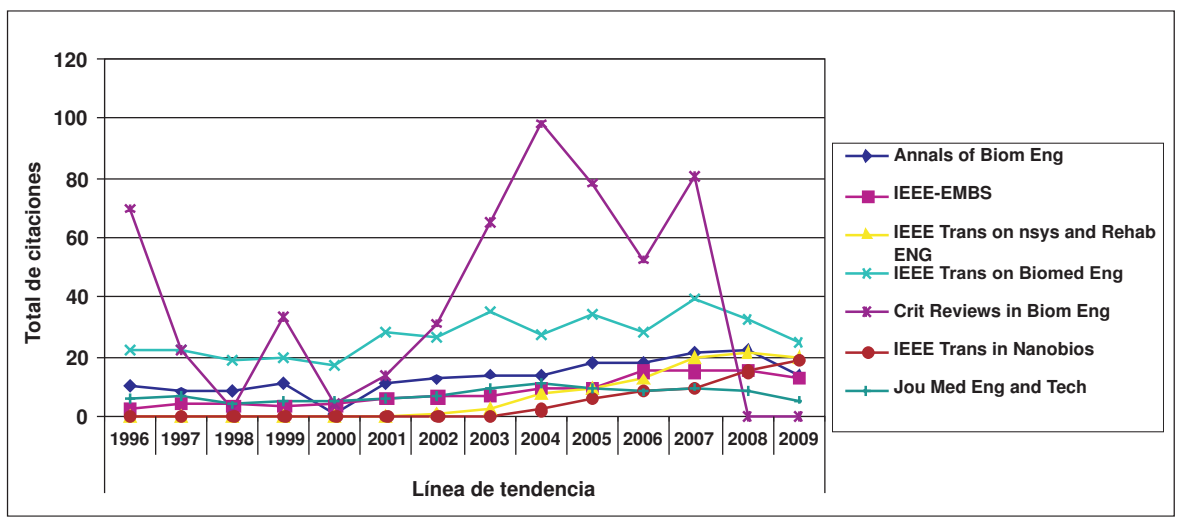

Figura 5. Línea de tendencia en publicaciones en algunas revistas de ingeniería biomédica, excepto las revistas Journal of Clinical Engineering y Biomedical Instrumentation \& Technology (16).

realizadas en el año, dividida por la cantidad de artículos publicados en ese mismo periodo.

A continuación veamos cómo se comportan estos indicadores en las revistas citadas anteriorment, en relación con los artículos científicos que tratan temas sobre ingeniería clínica.

La figura 2 muestra la cantidad de artículos que se citan en las revistas Journal of Clinical Engineering y Biomedical Instrumentation \& Technology.

Nótese que la tendencia es a la baja y que la cantidad de citaciones no supera las 60 en todo el período para la primera revista, y las 180, para la segunda, con valores promedios de 46,57 y 151,79 , respectivamente.

La figura 3 muestra el porcentaje de artículos que nunca son citados en las revistas Journal of Clinical Engineering y Biomedical Instrumentation \& Technology.

Nótese que la tendencia es al ascenso y que el porcentaje de artículos que nunca son citados alcanza el $90 \%$ para ambas revistas en los últimos cinco años, con valores promedios de $76,57 \%$ para la primera, y de $78,73 \%$, para la segunda.

La figura 4 muestra la línea de tendencia en el impacto de estas dos revistas.

Nótese que la tendencia es a la baja y que el índice de impacto en los últimos siete años bajó a un punto, alcanzando sus valores mínimos en 2008 con una valor por debajo de la unidad $(0,8)$. Se alcanzaron valores promedios de 1,92 unidades para la revista Journal of Clinical Engineering, y 1,52, para la revista Biomedical Instrumentation \& Technology.
Sólo como marco de referencia para que el lector tenga un nivel de comparación, en la figura 5 se muestra la línea de tendencia en publicaciones en algunas revistas de ingeniería biomédica, excepto las revistas Journal of Clinical Engineering y Biomedical Instrumentation \& Technology.

Nótese que la tendencia es al ascenso en algunos casos y, en otros, se mantiene constante o de manera sostenida, y que los valores en la tendencia están en el orden de 40 a 150 puntos.

Los anteriores hallazgos nos demuestran una verdad irrefutable: los temas tratados en las revistas de ingeniería clínica han perdido interés para la comunidad científica. Esto implica que hay que trabajar mucho más para posicionar la especialidad. Hay que usar las herramientas existentes en otras ramas del saber e integrarlas para resolver los problemas científicos que se tienen en ésta, esto es: investigación de operaciones, inteligencia artificial, modelos matemáticos, métodos multicriterios de toma de decisión, entre otros.

A continuación, se presenta un listado de los aspectos más relevantes en los cuales se puede trabajar en ingeniería clínica para que, desde un abordaje científico, se puedan resolver los problemas que aún persisten:

1) Adopción de la teoría de sistemas a la gestión tecnológica hospitalaria, viendo las funciones propias como procesos y estableciendo las relaciones entre éstos y las formas de medir su desempeño.

2) Establecer un marco común para la medición del desempeño de los programas de gestión tecnológica hospitalaria, en otras palabras, la aplicación del benchmarking en la especialidad. 
3) Aplicación de los métodos de ingeniería económica con el objetivo de realizar estudios de costo-beneficio.

4) Desarrollo de aplicaciones informáticas de alcance global que permitan establecer un marco común para el intercambio de información y evaluación de tecnologías, facilitando así la aplicación del benchmarking.

5) Aplicación de métodos de minería de datos, para evaluar el desempeño de los procesos asociados a la gestión tecnológica hospitalaria.

6) Aplicación de métodos de análisis de riesgos y probabilísticos de seguridad, establecidos en otras industrias, como la nuclear y la aeronáutica, a la tecnología médica compleja y de alto riesgo, para proveer una mayor seguridad a los pacientes.

7) Aplicación de los métodos de inteligencia artificial que asistan en la toma de decisión a la hora de evaluar y adquirir tecnologías y servicios.

No se puede decir que estos temas de investigación no estén siendo abordados. Lo que se está planteando acá es que los temas aún son insuficientes y que deben enfocarse con mayor rigor científico y aplicabilidad para que las experiencias sean tomadas como referencias, aumentando así el posicionamiento y la presencia de la especialidad en las publicaciones científicas.

\section{Conclusiones}

1. No existe un estudio riguroso que permita caracterizar el estado real de la ingeniería clínica en Colombia.

2. Al hacer un estudio del desarrollo de los grupos de investigación de ingeniería clínica en Colombia, se observa un desarrollo incipiente de la especialidad. Esto se evidencia en la baja calificación de los grupos debido a la escasez de su producción científica de alto nivel (proyectos y publicaciones).

3. Los temas tratados en las revistas científicas de ingeniería clínica han perdido interés para la comunidad científica. Esto se evidencia por el bajo número de citaciones que tienen los mismos, en comparación con otras publicaciones del mismo ramo.

4. El bajo número de citaciones y, por ende, el impacto de las investigaciones debe aumentarse aplicando herramientas existentes en otras ramas del saber e integrarlas a la ingeniería clínica, esto, con el objetivo de resolver los problemas científicos que se tienen en la especialidad.

\section{Conflictos de interés}

El autor de este trabajo deja constancia de que no existe conflicto de interés alguno.

\section{Financiación}

Los recursos para la financiación de este ensayo se obtuvieron gracias a COLCIENCIAS, convocatoria: 459/08 proyectos investigación prioritaria en salud.

\section{Referencias}

1. Grimes LS. The future of clinical engineering: The challenge of change. IEEE Eng Med Biol Mag. 2003:22;91-9.

2. Denis ER. Manual de ingeniería clínica. Puerto Ordaz: Fundacite; 2001. p. 1-210.

3. Kohn L, Corrigan MJ, Molla SD. Building a safer health system. To err is human. Washington, D.C.: National Academy Press; 2000. p. 1-8.

4. IMB. Autonomic computing: IBM perspective on the state of information technology. Armonk NY: International Bussiness Machines; 2001. p. 3-37. Fecha de consulta: 2 de junio 2009. Disponible en: http://www.research.ibm.com/ autonomic/manifesto/autonomic_computing.pdf.

5. Gibbs WW. Autonomic computing. Scientific American.com. Fecha de consulta: 2 de junio 2009. Disponible en: http:// www.scientificamerican.com/article.cfm?id=autonomiccomputing.

6. OECD. Health Data 2004: A comparative analysis of 30 countries. Washington, D.C.: OECD Publishing; 2004. p. $1-190$

7. Garrido UR. El gasto sanitario y su financiación: evolución, tendencias y sus reflexiones para el futuro. RevistaAsturiana de Economía. 2006:35;45-65. Fecha de consulta:2 de junio 2009. Disponible en: http://www.revistaasturianadeeconomia.org/ raepdf/35/P45URBANOS.pdf.

8. World Health Organization. World Health Report, 2000. Fecha de consulta: 2 de junio 2009. Disponible en: http:// www.who.int/whr/2000/en/report.htm

9. Whitaker Foundation. Biomedical Engineering Education Summit 2005. Fecha de consulta: 2 de junio 2009. Disponible en: http://bmes.seas.wustl.edu/WhitakerArchives/academic.

10. ACCE. Clinical Engineers: Stewards of Healthcare Technologies. Fecha de consulta: 2 de junio 2009. Disponible en: http://www.accenet.org/default.asp?page $=p$ ublications\&section=whitepapers.

11. ECRI. Health Technology. Special report on technology management: Preparing your hospital for the 1990s. Health Technol. 1989;3:1-68.

12. Asprilla CG. La crisis de los hospitales públicos en Colombia. Fecha de consulta: 2 junio 2009. Disponible en: http://www.voltairenet.org/article121511.html.

13. Ministerio de la Protección Social. Modelo de evaluación y gestión del equipamiento biomédico. Fecha de consulta: 2 junio 2009. Disponible en: http:// 
www.minproteccionsocial.gov.co/VBeContent/Library/ documents/DocNewsNo14710DocumentNo5095.pdf.

14. Colciencias. Base de Datos Scienti (Sistema de Información Científica Técnico de Colciencias). Fecha de consulta: 2 de junio 2009. Disponible en: http:// scienti.colciencias.gov.co:8081/digicyt.war/search/ EnGrupolnvestigacion/area.do?_tableAction=reset\&
tableName=enGrupolnvestigacion.table.area\&area conocimiento_cod_area_conhec $=31300006$.

15. Grove AS. Only the paranoid survive. New York: Doubleday Business; 1996. p. 12.

16. Scopus. Base de datos Scopus. Fecha de consulta: 5 de noviembre de 2009. Disponible en: http://www.scopus.com/ source/eval. 